\title{
Hemodynamic responses to strength exercise with blood flow restriction during different phases of the menstrual cycle
}

\author{
Gabriel Rodrigues Neto $1,2,3,4^{*}$, Jefferson Silva Novaes ${ }^{4,5}$, Adenilson Targino de Araújo \\ Júnior $^{2,6,7}$, Júlio César Gomes Silva ${ }^{1,2}$, Rodrigo Poderoso Souza ${ }^{2,7}$, Maria Socorro Cirilo- \\ Sousa ${ }^{1,2}$
}

\begin{abstract}
The present study aimed to determine the influence of low-load (LL) resistance exercise (RE) with blood flow restriction (BFR) on systolic blood pressure (SBP), diastolic blood pressure (DBP), mean blood pressure (MBP), heart rate (HR), double product (DP) and oxygen saturation (SpO2) during the phases of the menstrual cycle (MC). Thirty untrained women were randomly and proportionally divided into three groups: $\mathrm{HI}=$ high-intensity exercises $(80 \%$ of one-repetition maximum (1RM)); LL = low-load exercises $(20 \%$ of $1 \mathrm{RM})$; and LL+BFR = LL exercises combined with BFR. The exercise sessions were performed during the $3^{\text {rd }}-4^{\text {th }}$ days (follicular phase), $16^{\text {th }}$ day (ovulatory phase) and the $24^{\text {th }}-26^{\text {th }}$ days (luteal phase) of the MC. Before and immediately after the exercises, SBP, DBP, HR and SpO2 were evaluated. We observed an increase in SBP, HR and DP in the three phases of the MC for all groups $(p<$ 0.05). Groups LL and/or LL+BFR exhibited a greater increase in SBP, DBP, MBP, HR and DP when compared with the HI group $(p<0.05)$, and in the three groups, SpO2 was not reduced $(p>0.05)$. There was a significant effect of the MC phases on HR and DP $(p<0.05)$. We conclude that the three groups exhibited increased SBP, HR and DP; however, SpO2 was not different. Furthermore, groups LL and LL+BFR exhibited greater increases in hemodynamics, and the MC phases seem to influence only HR and DP.

Keywords: kaatsu training, vascular occlusion, women
\end{abstract}

\section{INTRODUCTION}

Women exhibit hormonal variations (estrogen and progesterone) during the three phases of the menstrual cycle (MC). These variations may promote a negative effect on cardiovascular responses due to the increased body temperature after ovulation and during the luteal phase (18-24 $4^{\text {th }}$ day of MC) (Jonge, 2003). In addition, it is well-established that differences exist in cardiovascular indicators between the sexes in response to exercise, and one of the influencing factors is the hormonal variation that occurs during the MC phases (O'Toole, 1988). Thus, some studies aimed to identify the influence of the MC on cardiovascular responses after different exercises (Hessemer \& Bruck, 1985; Lynn, McCord, \& Halliwill, 2007; Pivarnik, Marichal, Spillman, \& Morrow, 1992; Stachenfeld, Silva, \& Keefe, 2000). However, the influence of low-load resistance exercises (LL; $20-30 \%$ of one-repetition maximum (1RM)) combined with the blood flow restriction (BFR)

\footnotetext{
Manuscript received at November $5^{\text {th }} 2016$; Accepted at January $4^{\text {th }} 2017$

${ }^{1}$ Department of Physical Education, Graduate Associate Program in Physical Education, University of Pernambuco / Federal University of Paraiba - UPE / UFPB, João Pessoa, Paraíba, Brazil

${ }^{2}$ Department of Physical Education, Laboratory of Kinanthropometry and Human Performance

${ }^{3}$ Coordination of Physical Education, Nursing and Medical Schools, Nova Esperança (FAMENE/ FACENE)

${ }^{4}$ Department of Gymnastics, Federal University of Rio de Janeiro, Graduate Program in Physical Education, Rio de Janeiro, RJ, Brazil

${ }^{5}$ Department of Physical Education, Federal University of Juiz de Fora, Physical Education Post Graduation Program, Juiz de Fora, Brazil

${ }^{6}$ Federal Institute of Paraíba (IFPB), Department of Physical Education, Campina Grande, Paraíba - Brazil

${ }^{7}$ Department of Physical Education, University of Trás-os-Montes and Alto Douro (UTAD), Vila Real, Portugal

* Corresponding author: Coordination of Physical Education - Nursing and Medical Schools, Nova Esperança (FAMENE / FACENE). Avenida Frei Galvão, 12 - Gramame, PB, 58067-695, João Pessoa, Brazil. E-mail: gabrielrodrigues_1988@hotmail.com
} 
technique has not been investigated. This training method consists of using low weight (20-30\% of 1RM) combined with BFR promoted by elastic bands or standard sphygmomanometers (Sato, Yoshitomi, \& Abe, 2005).

The BFR training method has been used to increase strength (Laurentino et al., 2012; Silva et al., 2015; Vechin et al., 2015), muscle mass (Laurentino et al., 2012; Vechin et al., 2015), muscular endurance (Gil et al., 2017; Kacin \& Strazar, 2011), and functional capacity (Araujo et al., 2015) and was shown to be safe (Araújo et al., 2014; Neto et al., 2015; Neto et al., 2016; Takano et al., 2005). In this scenario, gains in strength and muscle mass with the use of this technique have been shown to be as effective as high-intensity resistance training (HI; $\geq 80 \%$ of 1RM). This contrasts with the position of the American College of Sports Medicine (ACSM), which recommends weights equal to or greater than $60 \%$ of $1 \mathrm{RM}$ to increase strength and muscle mass (ACSM, 2009). Additionally, only two studies analyzed the influence of this technique on strength and hypertrophy (Sakamaki, Yasuda, \& Abe, 2012) and muscular power and endurance (Gil et al., 2017) with respect to MC phases.

Some studies evaluated the acute effects of resistance exercises with BFR on systolic blood pressure (SBP), diastolic blood pressure (DBP), mean blood pressure (MBP), heart rate (HR), double product (DP) (Araújo et al., 2014; Neto et al., 2015; Neto et al., 2016; Okuno, Pedro, Leicht, Ramos, \& Nakamura, 2014; Rossow et al., 2012; Rossow et al., 2011; Takano et al., 2005; Vieira, Chiappa, Umpierre, Stein, \& Ribeiro, 2013) and oxygen saturation (SpO2) (Neto et al., 2016). However, no studies have evaluated these variables after sessions of resistance exercitation with BFR performed during the MC phases (follicular, ovulatory and luteal).

Thus, the first hypothesis of the present study was that LL performed with BFR would increase SBP, DBP, MBP, HR, DP and SpO2 values, similar to $\mathrm{HI}$ and LL without BFR. The second hypothesis was that the greatest increase in SBP, DBP, MBP, HR, DP and SpO2 values would occur in the luteal phase, when compared with the follicular and ovulatory phases. Therefore, the present study aimed to determine the influence of LL with BFR on SBP, DBP, MBP, $\mathrm{HR}, \mathrm{DP}$ and $\mathrm{SpO} 2$ during different $\mathrm{MC}$ phases.

\section{METHODS}

\section{Participants}

Thirty untrained women (age: $21.7 \pm 3.4$ years; body mass index: $23.5 \pm 3.9 \mathrm{~kg} \cdot \mathrm{m}^{-2}$ ) were randomly and proportionally divided into three groups: HI (exercises at $80 \%$ of $1 \mathrm{RM}$ ); LL (exercises at $20 \%$ of 1RM); and LL+BFR (LL combined with BFR). The sample size was determined using the software G*Power 3.1 (Faul, Erdfelder, Lang, \& Buchner, 2007), and based on an a priori analysis, we adopted a power of 0.80 , an $\alpha=0.05$, a correlation coefficient of 0.5 and an effect size of 0.50 ; therefore, $\mathrm{N}=30$ individuals was calculated. The sample size was sufficient to provide $81.7 \%$ of the statistical power. To calculate the sample, the procedures suggested by Beck (2013) were adopted.

Women with irregular menstrual cycles, with any type of ostheomyoarticular injury on the upper or lower limbs, those using contraceptives for at least six months, those under hormone replacement therapy and ingesting supplements and those who responded positively to any of the items of the Physical Activity Readiness Questionnaire/PAR-Q were excluded from the study (Shephard, 1988). After the risks and benefits of the survey were explained, the participants signed the informed consent form prepared according to the Helsinki Statement. The study was approved by the Human Ethics and Research Committee of the Federal University of Paraíba (Universidade Federal da Paraíba) (State of João Pessoa), under protocol 0476/13.

\section{Study design}

On the first visit ( $1^{\text {st }}$ day of the follicular phase), an anthropometric evaluation was conducted, the BFR point was determined, and the maximum strength was evaluated using the 1RM test. Subsequently, the volunteers were subjected to three training sessions (biceps curl 
and knee extension). Those sessions occurred during the $3^{\text {rd }}-4^{\text {th }}$ days (follicular phase), $16^{\text {th }}$ day (ovulatory phase) and $24^{\text {th }}-26^{\text {th }}$ days (luteal phase) of the MC. On the $14^{\text {th }}$ day (ovulatory phase), the maximum strength was evaluated to adjust the load. Before and immediately after the exercises, SBP, DBP, $\mathrm{HR}$ and $\mathrm{SpO} 2$ were evaluated (Figure 1). The three groups in the study performed the following routine: HI; LL; and LL+BFR.

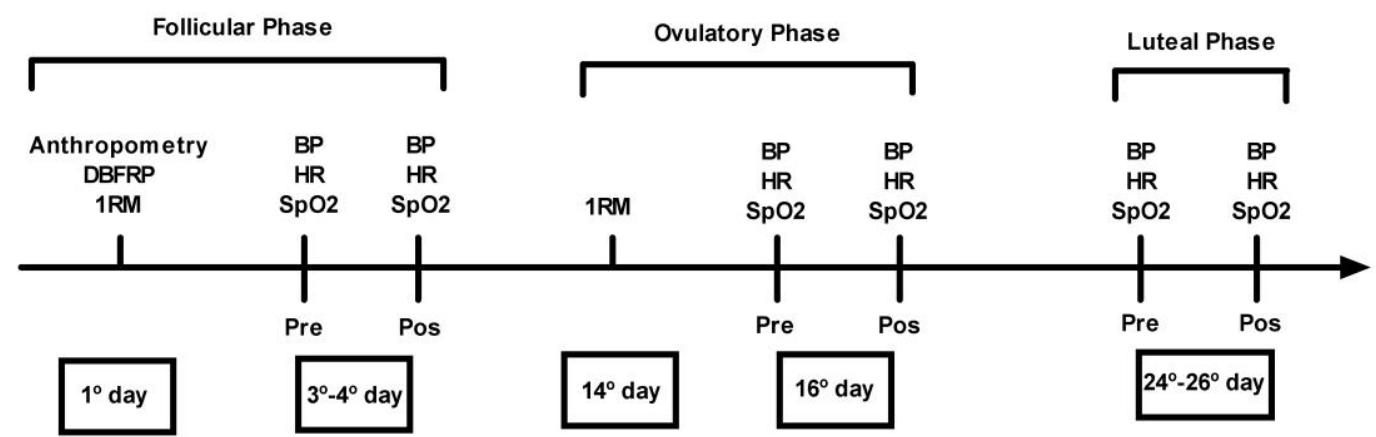

Figure 1. Experimental design. Note: DBFRP = determination of blood flow restriction point; $\mathrm{BP}=\mathrm{blood}$ pressure; $\mathrm{HR}=$ heart rate; $\mathrm{SpO} 2$ = oxygen saturation.

\section{Procedures}

Anthropometric evaluation

Height and body mass were measured with a precision of $0.5 \mathrm{~cm}$ and $0.1 \mathrm{~kg}$, respectively, using a stadiometer and a Filizola ${ }^{\circledR}$ scale. These measurements were used to obtain the body mass index $\left(\mathrm{kg} \cdot \mathrm{m}^{-2}\right)$.

\section{Blood flow restriction determination}

Total blood flow restriction was performed using vascular Doppler (MedPeg® DV -2001, Ribeirão Preto, State of São Paulo - SP, Brazil), in which the probe was placed on the radial artery (arms) and on the tibial artery (legs) to determine the arterial pressure $(\mathrm{mmHg})$ of BFR. The participants stayed in the supine position, and a standard blood pressure sphygmomanometer (pneumatic tourniquet komprimeter to hemostasis in extremities Riester) for biceps (width $60 \mathrm{~mm}$; length 470 $\mathrm{mm}$ ) and for knee extension (width $100 \mathrm{~mm}$; length $540 \mathrm{~mm}$ ) was placed on the axillary and inguinal fold regions, respectively, and was inflated until the auscultation pulse of the radial and tibial artery was interrupted. The cuff pressure used during the exercises was set to be $80 \%$ of the pressure needed for total blood flow restriction at rest (Laurentino et al., 2012). The cuff was deflated between sets.
One-Repetition Maximum (1RM) Testing

The prescription of the training load was evaluated using a 1RM test (ACSM, 2000). The evaluations were performed during the $1^{\text {st }}$ day (follicular phase) and the $14^{\text {th }}$ day (ovulatory phase), and the load was adjusted immediately after each evaluation. The exercises were performed bilaterally: direct biceps curl and knee extension. For recovery time between exercises, a standard of 10 minutes was used. The warmup consisted on two series of 5-10 repetitions at $40-60 \%$ (one-minute interval between the series) of the maximum perception of the strength of the individual. After a one-minute interval, the third series was concluded using 35 repetitions at $60-80 \%$ of the maximum perceived strength. After one more rest period (1 min), the strength evaluation was started, in which up to five attempts could be performed, adjusting the load before every new attempt. The recovery duration between the attempts was standardized at 3-5 minutes. The test was interrupted when the individual could not perform the movement correctly, and the repetition with complete execution was considered the maximum load.

Blood Pressure (BP), Heart Rate (HR) and Double Product (DP)

The participants were equipped with an automatic blood pressure monitor (OMROM; model HEM-705CP) (Vera-Cala, Orostegui, 
Valencia-Angel, López, \& Bautista, 2011). The cuff was placed on the right arm, which was completely surrounded, covering at least twothirds of the upper region of the arm. This device was used for all pre- and post-session systolic and diastolic blood pressure measurements. All measurements were performed according to guidelines of the American Heart Association (Pickering et al., 2005). The HR was continuously monitored preand post-sessions (Polar T31 codedTM transmitter). DP was obtained by multiplying the HR (bpm) by the SBP (mm Hg). MBP was calculated using the equation $(\mathrm{SBP}+2 \mathrm{DBP}) / 3$.

\section{Oxygen Saturation (SpO2) Level}

The oxygen saturation (SpO2) level was evaluated using a finger oximeter (model CMS50DL; OXYM2000) at the pre- and postsession moments.

\section{Exercise protocol}

Each group (HI, LL and LL+BFR) performed two resistance exercises bilaterally: direct biceps curl (with a conventional bar and discs) and knee extension (leg extension). In the HI group, participants completed four series of eight repetitions with $80 \%$ of $1 \mathrm{RM}$ with two-minute intervals between series and one minute between exercises. In the LL group, participants completed a series of 30 repetitions followed by three series of 15 repetitions using $20 \%$ of 1RM with 30 seconds of rest between all series and one minute between exercises. In the LL+BFR group, participants performed the same series, repetitions and rest as those in LL while using a standard blood pressure sphygmomanometer for BFR. The pressure of the cuff was maintained throughout the exercise session, except for the 30-second intervals between the series. The execution speed was established at four seconds (two seconds for concentric and two seconds for eccentric muscular action), controlled by the metronome.

\section{Statistical analysis}

The statistical analysis was initially performed using the Shapiro-Wilk normality test, the Levene homogeneity test and Mauchly's sphericity test. The variables exhibited normal distribution or homogeneity or sphericity $(p>$ 0.05). Three-way repeated measures ANOVA (Group [HI vs. LL vs. LL+BFR] $\times$ Time [Pre-test vs. Post-test] $\times$ Phases [Follicular vs. Ovulatory vs. Luteal]) was used, and a post hoc Bonferroni test was used to analyze possible differences in the dependent variables. The effect size (ES) was used to determine the magnitude (trivial $<0.50$, small $=0.50-1.25$, moderate $=1.25-1.9$, large $>$ 2.0) of changes between the evaluations of the study protocols (Rhea, 2004). The significance level was set at $p<0.05$. All statistical analyses were performed using the statistical software package SPSS version 20.0 (SPSS Inc., Chicago, IL).

\section{RESULTS}

The mean pressure used throughout the exercise protocol was as follows: arms, right $=$ $129.0 \pm 11.0 \mathrm{mmHg}$, left $=128.0 \pm 13.9$ $\mathrm{mmHg}$; and legs, right $=136.0 \pm 9.6 \mathrm{mmHg}$, left $=136.0 \pm 12.6 \mathrm{mmHg}$.

\section{Systolic Blood Pressure (SBP)}

There was no interaction among group $x$ time $\times$ phases or between group $\times$ time, group $\times$ phases, or time $\times$ phases $(p>0.05)$. There were no significant effects of phases $(p=0.430)$; however, significant differences were observed between groups $(p=0.003)$ and time $(p<$ 0.001). With respect to the group effect, significant differences were observed between the HI vs. LL groups and the HI vs. LL+BFR groups ( $p<0.001 ; p=0.006$, respectively) in the ovulatory phase and between the HI vs. LL groups $(p=0.017)$ in the luteal phase. With respect to the time effect, we observed an increase in the three phases of the MC for all groups $(p<0.05)$, as shown in Table 1 .

\section{Diastolic Blood Pressure (DBP)}

There was no interaction among group $x$ time $\times$ phases or between group $\times$ time or time $\times$ phases $(p>0.05)$; however, there was a significant interaction between group $\times$ phases $(p=0.040)$. There were no significant effects between the phases $(p=0.889)$ or the time $(p=$ $0.355)$; however, significant effects were 
observed between groups $(p<0.001)$. With respect to the group effect, significant differences were observed between the $\mathrm{HI}$ and LL groups and the HI and LL+BFR groups ( $p=$ 0.006; $p=0.044$, respectively) in the follicular phase, between the HI and LL groups and the HI and LL+BFR groups $(p<0.001 ; p=0.001$, respectively) in the ovulatory phase, and between the $\mathrm{HI}$ and LL groups and the HI and LL+BFR groups $(p=0.015 ; p<0.001$, respectively) in the luteal phase, as shown in Table 1.

\section{Mean Blood Pressure (MBP)}

There was no interaction among group $x$ time $\times$ phases or between group $\times$ time or time $\times$ phases $(p>0.05)$; however, there was a significant interaction between group $\times$ phases $(p=0.011)$. There were no significant effects between phases $(p=0.919)$, but significant differences were observed between groups $(p<$ $0.001)$ and times $(p<0.001)$. With respect to the group effect, significant differences were observed between the HI and LL groups ( $p=$ 0.013) in the follicular phase, between the HI and LL groups and the HI and LL+BFR groups $(p<0.001 ; p<0.001$, respectively) in the ovulatory phase, and between the HI and LL groups and the HI and LL+BFR groups ( $p=$ 0.003; $p<0.001$, respectively) in the luteal phase. With respect to the time effect, we observed an increase in the three phases of the MC for the LL+BFR group $(p<0.05)$ and in the ovulatory and luteal phases for the LL group ( $p$ $<0.05$ ), as shown in Table 1 .

\section{Heart Rate (HR)}

There was no interaction among group $x$ time $\times$ phases or between group $x$ phases or time $\times$ phases $(p>0.05)$; however, there was a significant interaction between group $\times$ time $(p$ $<0.001)$. There were significant effects between groups $(p=0.033)$, phases $(p=0.001)$ and times $(p<0.001)$. With respect to the group effect, significant differences were observed between the HI and LL groups and the LL and LL+BFR groups $(p<0.001 ; p<0.001$, respectively) in the follicular phase, between the $\mathrm{HI}$ and LL groups and the LL and LL+BFR groups ( $p=0.002 ; p<0.001$, respectively) in the ovulatory phase, and between the HI and LL groups and the LL and LL+BFR groups $(p<$ $0.001 ; p<0.001$, respectively) in the luteal phase. With respect to the phases effect, significant differences were observed between the follicular and ovulatory phases and the follicular and luteal phases $(p<0.001 ; p=$ 0.019 , respectively) in the LL group. With respect to the time effect, we observed an increase in the three phases of the MC for the HI and LL+BFR groups $(p<0.05)$ and in the follicular phase for the LL group ( $p=0.006)$, as shown in Table 1.

\section{Double Product (DP)}

There was no interaction among group $x$ time $\times$ phases or between group $\times$ time, group $\times$ phases, or time $\times$ phases $(p>0.05)$. There were significant effects among groups $(p<$ $0.05)$, phases $(p=0.006)$ and times $(p<0.001)$. With respect to the group effects, significant differences were observed between the $\mathrm{HI}$ and LL groups and the HI and LL+BFR groups ( $p=$ 0.014; $p=0.019$, respectively) in the ovulatory phase and between the HI and LL groups and the HI and LL+BFR groups $(p=0.010 ; p=$ 0.022 , respectively) in the luteal phase. With respect to the phases effect, significant differences were observed between the follicular and ovulatory phases and the follicular and luteal phases $(p=0.034 ; p=0.020$, respectively) in the LL group and between the follicular and ovulatory phases and the follicular and luteal phases $(p=0.027 ; p=0.028$, respectively) in the $\mathrm{LL}+\mathrm{BFR}$ group. With respect to the time effect, we observed an increase in the three phases of the MC for all groups $(p<0.05)$, as shown in Table 1 .

\section{Oxygen Saturation (Sp02)}

There was no interaction among group $x$ time $\times$ phases or between group $\times$ time or time $\times$ phases $(p>0.05)$; however, there was a significant interaction between group $\times$ phases $(p=0.013)$. There were no significant effects among groups $(p=0.344)$, phases $(p=0.302)$ or time $(p=0.122)$, as shown in Table 1 . 
Table 1

Comparative analysis of systolic blood pressure (SBP), diastolic blood pressure (DBP), mean blood pressure (MBP), heart rate (HR), double product (DP) and oxygen saturation (SpO2) among the study groups.

\begin{tabular}{|c|c|c|c|c|c|c|}
\hline \multirow{2}{*}{ SBP (mmHg) } & \multicolumn{2}{|c|}{ Follicular Phase } & \multicolumn{2}{|c|}{ Ovulatory Phase } & \multicolumn{2}{|c|}{ Luteal Phase } \\
\hline & Pre-test & Post-test & Pre-test & Post-test & Pre-test & Post-test \\
\hline HI & $100.1 \pm 13.8$ & $125.0 \pm 11.4^{*}$ & $100.1 \pm 9.5$ & $117.6 \pm 12.2^{*}$ & $102.6 \pm 13.0$ & $121.9 \pm 13.9^{*}$ \\
\hline LL & $113.3 \pm 4.2$ & $133.1 \pm 8.4^{*}$ & $110.1 \pm 7.9$ & $138.7 \pm 7.2^{* * * *}$ & $110.2 \pm 5.2$ & $136.9 \pm 10.2^{* * * *}$ \\
\hline $\mathrm{LL}+\mathrm{BFR}$ & $105.1 \pm 10.1$ & $129.2 \pm 16.6^{*}$ & $106.1 \pm 11.9$ & $133.4 \pm 20.5^{*} \dagger$ & $109.4 \pm 9.4$ & $133.9 \pm 23.0^{*}$ \\
\hline \multirow{2}{*}{ DBP (mmHg) } & \multicolumn{2}{|c|}{ Follicular Phase } & \multicolumn{2}{|c|}{ Ovulatory Phase } & \multicolumn{2}{|c|}{ Luteal Phase } \\
\hline & Pre-test & Post-test & Pre-test & Post-test & Pre-test & Post-test \\
\hline HI & $65.9 \pm 6.5$ & $62.3 \pm 7.1$ & $59.7 \pm 10.0$ & $59.1 \pm 8.5$ & $63.3 \pm 9.6$ & $60.6 \pm 9.3$ \\
\hline LL & $71.8 \pm 8.7$ & $71.8 \pm 9.2^{* *}$ & $70.3 \pm 5.9$ & $75.7 \pm 10.0^{* *}$ & $68.4 \pm 8.7$ & $70.3 \pm 4.7^{* *}$ \\
\hline $\mathrm{LL}+\mathrm{BFR}$ & $66.9 \pm 6.3$ & $69.2 \pm 6.1 \dagger$ & $71.4 \pm 9.4$ & $74.3 \pm 13.9 \dagger$ & $68.1 \pm 5.6$ & $75.8 \pm 11.7 \dagger$ \\
\hline \multirow{2}{*}{ MBP (mmHg) } & \multicolumn{2}{|c|}{ Follicular Phase } & \multicolumn{2}{|c|}{ Ovulatory Phase } & \multicolumn{2}{|c|}{ Luteal Phase } \\
\hline & Pre-test & Post-test & Pre-test & Post-test & Pre-test & Post-test \\
\hline HI & $77.3 \pm 8.1$ & $83.2 \pm 8.3$ & $73.1 \pm 9.1$ & $78.6 \pm 8.5$ & $76.4 \pm 9.6$ & $81.0 \pm 9.1$ \\
\hline LL & $85.6 \pm 6.8$ & $92.2 \pm 7.3^{* *}$ & $83.5 \pm 5.2$ & $96.2 \pm 7.2^{* * * *}$ & $82.3 \pm 4.9$ & $92.5 \pm 5.8^{* * * *}$ \\
\hline $\mathrm{LL}+\mathrm{BFR}$ & $79.6 \pm 7.3$ & $89.2 \pm 8.8^{*}$ & $82.9 \pm 9.1$ & $94.0 \pm 13.2^{*} \dagger$ & $81.8 \pm 5.6$ & $95.1 \pm 12.3^{*} \dagger$ \\
\hline \multirow{2}{*}{ HR Bpm } & \multicolumn{2}{|c|}{ Follicular Phase } & \multicolumn{2}{|c|}{ Ovulatory Phase } & \multicolumn{2}{|c|}{ Luteal Phase } \\
\hline & Pre-test & Post-test & Pre-test & Post-test & Pre-test & Post-test \\
\hline HI & $80.5 \pm 5.2$ & $107.2 \pm 17.9^{*}$ & $84.5 \pm 9.4$ & $111.7 \pm 13.5^{*}$ & $83.0 \pm 6.8$ & $108.7 \pm 9.3^{*}$ \\
\hline LL & $75.2 \pm 9.9$ & $111.7 \pm 11.6^{*}, * *$ & $91.1 \pm 10.4$ & $118.5 \pm 13.1^{* * * *} ¥$ & $85.1 \pm 15.2$ & $120.5 \pm 13.4^{* * * *+\$} \mathbb{S}$ \\
\hline $\mathrm{LL}+\mathrm{BFR}$ & $78.9 \pm 8.5$ & $112.4 \pm 22.5^{*,+}+$ & $80.6 \pm 11.4$ & $119.7 \pm 25.9^{*+*}$ & $78.8 \pm 10.5$ & $119.2 \pm 22.5^{*}$ \\
\hline \multirow{2}{*}{$\mathrm{DP}$ bpm*mmHg } & \multicolumn{2}{|c|}{ Follicular Phase } & \multicolumn{2}{|c|}{ Ovulatory Phase } & \multicolumn{2}{|c|}{ Luteal Phase } \\
\hline & Pre-test & Post-test & Pre-test & Post-test & Pre-test & Post-test \\
\hline HI & $8029.7 \pm 989.4$ & $13519.0 \pm 3170.2^{*}$ & $8478.0 \pm 1328.4$ & $13229.7 \pm 2726.2^{*}$ & $8525.9 \pm 1400.1$ & $13311.4 \pm 2306.0^{*}$ \\
\hline LL & $8517.4 \pm 1169.6$ & $14910.6 \pm 2575.8^{*}$ & $10029.0 \pm 1335.9$ & $16462.7 \pm 2229.7^{*, * *, ¥}$ & $9412.6 \pm 1921.4$ & $16565.2 \pm 2769.6^{*, * *, \mathbb{S}}$ \\
\hline $\mathrm{LL}+\mathrm{BFR}$ & $8274.5 \pm 1023.2$ & $14661.4 \pm 4036.3^{*}$ & $8495.7 \pm 1152.8$ & $16280.7 \pm 5555.3^{*}+\ngtr$ & $8592.2 \pm 1160.4$ & $16210.7 \pm 5010.6^{*} \uparrow \$$ \\
\hline \multirow{2}{*}{$\mathrm{SpO} 2(\%)$} & \multicolumn{2}{|c|}{ Follicular Phase } & \multicolumn{2}{|c|}{ Ovulatory Phase } & \multicolumn{2}{|c|}{ Luteal Phase } \\
\hline & Pre-test & Post-test & Pre-test & Post-test & Pre-test & Post-test \\
\hline HI & $97.9 \pm 0.9$ & $98.0 \pm 1.0$ & $97.4 \pm 1.0$ & $97.9 \pm 1.1$ & $97.0 \pm 0.9$ & $95.7 \pm 4.6$ \\
\hline LL & $98.2 \pm 0.7$ & $97.7 \pm 0.9$ & $97.9 \pm 0.9$ & $96.5 \pm 1.9$ & $98.3 \pm 0.8$ & $98.0 \pm 0.8$ \\
\hline LL+BFR & $97.4 \pm 1.9$ & $96.7 \pm 2.9$ & $97.7 \pm 1.1$ & $97.5 \pm 0.9$ & $97.4 \pm 1.5$ & $96.6 \pm 2.4$ \\
\hline
\end{tabular}

Note: $\mathrm{HI}=$ high-intensity group; $\mathrm{LL}=$ low-load group; LL+BFR $=$ low-load group + blood flow restriction. ${ }^{*}$ significant difference between pre-test and post-test; ${ }^{* *}$ significant difference between the HI and LL groups; $\dagger$ significant difference between the HI and LL+BFR groups; $\$$ significant difference between the LL and LL+BFR groups; $¥$ significant difference between the follicular and ovulatory phases; $\mathbb{S}$ significant difference between the follicular and luteal phases

\section{DISCUSSION}

The present study investigated the influence of LL with BFR on SBP, DBP, MBP, HR, DP and SpO2 during different $\mathrm{MC}$ phases. To our knowledge, the present study is the first to evaluate the effect of resistance training with BFR on hemodynamics with respect to MC phases in women. The primary results of the present study were as follows: i) the three groups exhibited increased SBP, HR and DP but did not exhibit differences on SpO2; ii) the LL and LL+BFR treatments resulted in greater hemodynamic increases compared with the HI treatment; and iii) the phases of the MC seem to influence only HR and DP. Nonetheless, we observed a significant increase in SBP, HR and DP among the three exercise groups, and between the few studies that evaluated these variables after RE with BFR (Araújo et al., 2014; Brandner, Kidgell, \& Warmington, 2015; Neto et al., 2016; Okuno et al., 2014), two corroborate with our findings (Brandner et al., 2015; Neto et al., 2016). These authors reported increased SBP, DBP and MBP (Brandner et al., 2015) and HR and DP (Brandner et al., 2015; Neto et al., 2016) immediately after LL with BFR and after LL and HI exercises. Thus, we concluded that the increased values observed in the present study are within the normal patterns, which reinforces the safety of both continuous and intermittent BFR (Brandner et al., 2015).

With respect to the greater increase in hemodynamics in the LL and LL+BFR groups when compared with the HI group, four studies disagree with our findings (Fahs et al., 2011; Okuno et al., 2014; Poton \& Polito, 2016, 2015), and one corroborates with our findings (Araújo et al., 2014). In this context, we observed that the hemodynamics seem to significantly increase the HI protocol when compared with LL or 
$\mathrm{LL}+\mathrm{BFR}$ in men. However, this increase seems to occur differently in women, as observed in the present study and in the study by Araújo et al. (2014), which was the only study to evaluate only women. Thus, we speculate that women may be more sensitive to the accumulation of metabolites and the intramuscular $\mathrm{pH}$ reduction caused by BFR, which may cause more discomfort and thus increase the hemodynamics for both healthy and hypertensive women (Araújo et al., 2014).

With respect to $\mathrm{SpO} 2$, our results corroborate the findings of Neto et al. (2016). Although there were no significant differences, we observed a little reduction for the LL and LL+BFR groups in the three phases of the MC. Neto et al. (2016) reported a reduction in $\mathrm{SpO} 2$ for the LL and LL+BFR protocols and mentioned that this reduction occurred because training with BFR decreases the amount of oxygen transported by the blood, perhaps caused by the blood occlusion. Consequently, there would be a lower availability of $\mathrm{O} 2$ for consumption in the muscle tissue. Thus, it is likely that a HI resistance training is able to promote greater blood mobilization (Copeland et al., 1996) and thus a greater influx of postexercise muscle oxygen when compared with LL training, irrespective of BFR status, which does not reduce $\mathrm{SpO} 2$.

Although no articles evaluating the hemodynamics of women monitored the MC after resistance exercises, one study reported a significant increase in HR in the luteal phase when compared with the follicular phase after aerobic exercise (Pivarnik et al., 1992). Our findings are in accordance and we could speculate that this may have also occurred with DP. Thus, the hormone variation and the changes in the estrogen (anabolic effect) and progesterone (catabolic effect) levels during the MC phases may affect HR and DP (Jonge, 2003). In addition, although no study has evaluated the effect of LL with BFR on hemodynamics during the MC phases, two studies evaluated the effect of resistance exercises with BFR with respect to the phases of the MC (Gil et al., 2017; Sakamaki et al., 2012).
Gil et al. (2017) analysed the effect of strength training with BFR on muscular power and endurance in the three phases of the MC. The authors concluded that BFR does not seem to increase the power of the upper and lower limbs, but it may be a good strategy to improve muscular endurance, especially in the ovulatory and luteal phase of the MC. In turn, Sakamaki et al. (2012) compared the effect of LL combined with BFR on muscle hypertrophy and strength during the follicular and luteal phases of the MC. The results indicated that increased muscle hypertrophy and strength were greater in the luteal phase than in the follicular phase. Sakamaki et al. (2012) also examined the influence of estradiol, progesterone and testosterone on muscle strength and hypertrophy, aiming to analyse the possible changes resulting from hormone variations that occur during the MC. Interestingly, the authors observed that the hormones did not influence the increased muscle strength and hypertrophy. Thus, as hormones did not influence strength and hypertrophy, we speculate that in the present study, hormones did not influence SBP, DBP, MBP and SpO2. However, we can observe that even though the hormone variation did not influence strength and hypertrophy in the study by Sakamaki et al. (2012), in both studies, the luteal phase was the only phase with greater increases in muscular endurance (Gil et al., 2017), strength and hypertrophy (Sakamaki et al., 2012). In the present study, the increase in the HR and DP demonstrates the need for attention that health professionals and trainers must pay to the MC phases of women, with an emphasis on the luteal phase.

Thus, to prevent possible limitations of hemodynamic variations during the experiment, the present study focused on evaluating hemodynamics with respect to the three phases of the MC (follicular, ovulatory and luteal), which reflects the originality and relevance of the present study. However, with respect to the results obtained in the present study, some limiting factors became relevant, for example, the levels of endothelium-dependent vasodilator agents, autonomic nervous activity and cardiac debt. In addition, hormonal variation was not 
determined (estrogen, progesterone) to detect the possible influences that occur during the MC phases.

\section{CONCLUSIONS}

In conclusion, the three treatments increased SBP, HR and DP but did not change SpO2. The LL and LL+BFR treatments promoted a greater increase in hemodynamics compared with the $\mathrm{HI}$ treatment, and the MC phases influenced only HR and DP. Thus, it is important to conduct new experiments that analyse the hemodynamics in women with different levels of physical conditioning, especially involving other exercises and other intensities using BFR.

Acknowledgments:

Nothing to declare

Conflict of interest:

Nothing to declare

Funding:

Nothing to declare

\section{REFERÊNCIAS}

ACSM. (2000). American College of Sports Medicine. ACSM's Guidelines for Exercise Testing and Prescription (6th ed ed.). Baltimore, MD: Williams \& Wilkins.

ACSM. (2009). American College of Sports Medicine. Progression models in resistance training for healthy adults. Medicine and Science in Sports and Exercise, $\quad 41(3), \quad 687-708 . \quad$ doi: 10.1249/MSS.0b013e3181915670

Araujo, J. P., Neto, G. R., Loenneke, J. P., Bemben, M. G., Laurentino, G., Batista, G., . . Cirilo-Sousa, M. S. (2015). The effects of water aerobics in combination with blood flow restriction on strength and functional capacity in postmenopausal women. AGE. doi: 10.1007/s11357015-9851-4

Araújo, J. P., Silva, E. D., Silva, J. C. G., Souza, T. S. P., Lima, E. O., Guerra, I., \& Sousa, M. S. C. (2014). The acute effect of resistance exercise with blood flow restriction with hemodynamic variables on hypertensive subjects. Journal of Human Kinetics, 43(1), 79-85. doi: 10.2478/hukin-2014-0092
Beck, T. W. (2013). The importance of a priori sample size estimation in strength and conditioning research. Journal of Strength and Conditioning Research, 27(8), 2323-2337. doi: 10.1519/JSC.0b013e318278eea0

Brandner, C. R., Kidgell, D. J., \& Warmington, S. A. (2015). Unilateral bicep curl hemodynamics: Low-pressure continuous vs high-pressure intermittent blood flow restriction. Scandinavian Journal of Medicine and Science in Sports, 25(6), 770-777. doi: 10.1111/sms.12297

Copeland, S. R., Mills, M. C., Lerner, J. L., Crizer, M. F., Thompson, C. W., \& Sullivan, J. M. (1996). Hemodynamic effects of aerobic vs resistance exercise. Journal of Human Hypertension, 10(11), 747-753.

Fahs, C. A., Rossow, L. M., Seo, D., Loenneke, J. P., Sherk, V. D., Kim, E., . . Bemben, M. G. (2011). Effect of different types of resistance exercise on arterial compliance and calf blood flow. European Journal of Applied Physiology, 111 (12), 2969-2975. doi: 10.1007/s00421-011-1927-y

Faul, F., Erdfelder, E., Lang, A. G., \& Buchner, A. (2007). G* Power 3: A flexible statistical power analysis program for the social, behavioral, and biomedical sciences. Behavior Research Methods, 39(2), 175-191.

Gil, A. L. S., Neto, G. R., Sousa, M. S. C., Dias, I., Vianna, J., Nunes, R. A. M., \& Novaes, J. S. (2017). Effect of strength training with blood flow restriction on muscle power and submaximal strength in eumenorrheic women. Clinical Physiology and Functional Imaging, 37(2), 221-228. doi: 10.1111/cpf.12291

Hessemer, V., \& Bruck, K. (1985). Influence of menstrual cycle on thermoregulatory, metabolic, and heart rate responses to exercise at night. Journal of Applied Physiology, 59(6), 1911-1917.

Janse de Jonge, X. A. K. (2003). Effects of the menstrual cycle on exercise performance. Sports Medicine, 33(11), 833-851.

Kacin, A., \& Strazar, K. (2011). Frequent low-load ischemic resistance exercise to failure enhances muscle oxygen delivery and endurance capacity. Scandinavian Journal of Medicine and Science in Sports, 21(6), 231-241. doi: 10.1111/j.16000838.2010.01260.x

Laurentino, G. C., Ugrinowitsch, C., Roschel, H., Aoki, M. S., Soares, A. G., Neves, M., ... Tricoli, V. (2012). Strength training with blood flow restriction diminishes myostatin gene expression. Medicine and Science in Sports and Exercise, 44(3), 406-412. 
https://doi.org/10.1249/MSS.0b013e318233b4b c

Lynn, B. M., McCord, J. L., \& Halliwill, J. R. (2007). Effects of the menstrual cycle and sex on postexercise hemodynamics. American Journal of Physiology-Regulatory, Integrative and Comparative Physiology, 292(3), R1260-R1270. doi: 10.1152/ajpregu.00589.2006

Neto, G. R., Sousa, M. S. C., Costa, P. B., Salles, B. F., Novaes, G. S., \& Novaes, J. S. (2015). Hypotensive effects of resistance exercises with blood flow restriction. Journal of Strength and Conditioning Research, 29(4), 1064-1070. doi: 10.1519/JSC.0000000000000734

Neto, G. R., Sousa, M. S. C., Silva, G. V. C., Gil, A. L. S., Salles, B. F., \& Novaes, J. S. (2016). Acute resistance exercise with blood flow restriction effects on heart rate, double product, oxygen saturation and perceived exertion. Clinical Physiology and Functional Imaging, 36(1), 53-59. doi: $10.1111 /$ cpf.12193

O'Toole, M. L. (1988). Gender differences in the cardiovascular response to exercise. Cardiovascular clinics, 19(3), 17-33.

Okuno, N. M., Pedro, R. E., Leicht, A. S., Ramos, P. S., \& Nakamura, F. Y. (2014). Cardiac autonomic recovery after a single session of resistance exercise with and without vascular occlusion. Journal of Strength and Conditioning Research, 27(9), 1143-1150. doi: 10.1519/JSC.0000000000000245

Pickering, T. G., Hall, J. E., Appel, L. J., Falkner, B. E., Graves, J., Hill, M. N., . . Roccella, E. J. (2005). Recommendations for blood pressure measurement in humans and experimental animals part 1: blood pressure measurement in humans: a statement for professionals from the Subcommittee of Professional and Public Education of the American Heart Association Council on High Blood Pressure Research. Circulation, 111(5), 697-716.

Pivarnik, J. M., Marichal, C. J., Spillman, T., \& Morrow, J. R. (1992). Menstrual cycle phase affects temperature regulation during endurance exercise. Journal of Applied Physiology, 72 (2), 543548.

Poton, R., \& Polito, M. D. (2016). Hemodynamic response to resistance exercise with and without blood flow restriction in healthy subjects. Clinical Physiology and Functional Imaging, 36(3), 231-236. doi: $10.1111 /$ cpf.12218

Poton, R., \& Polito, M. D. (2015). Hemodynamics responses during lower-limb resistance exercise with blood flow restriction in healthy subjects.
Journal of Sports Medicine and Physical Fitness, 55(12), 1571-1577.

Rhea, M. R. (2004). Determining the magnitude of treatment effects in strength training research through the use the effect size. Journal of Strength and Conditioning Research, 18(4), 918-920.

Rossow, L. M., Fahs, C. A., Loenneke, J. P., Thiebaud, R. S., Sherk, V. D., Abe, T., \& Bemben, M. G. (2012). Cardiovascular and perceptual responses to blood-flow-restricted resistance exercise with differing restrictive cuffs. Clinical Physiology and Functional Imaging, 32(5), 331-337. doi: 10.1111/j.1475-097X.2012.01131.x

Rossow, L. M., Fahs, C. A., Sherk, V. D., Seo, D., Bemben, D. A., \& Bemben, M. G. (2011). The effect of acute blood-flow-restricted resistance exercise on postexercise blood pressure. Clinical Physiology and Functional Imaging, 31 (6), 429-434. doi: 10.1111/j.1475-097X.2011.01038.x

Sakamaki, M., Yasuda, T., \& Abe, T. (2012). Comparison of low-intensity blood flowrestricted training-induced muscular hypertrophy in eumenorrheic women in the follicular phase and luteal phase and agematched men. Clinical Physiology and Functional Imaging, 32(1), 185-191. doi: 10.1111/j.1475097X.2011.01075.x

Sato, Y., Yoshitomi, A., \& Abe, T. (2005). Acute growth hormone response to low-intensity KAATSU resistance exercise: comparison between arm and leg. International Journal of KAATSU Training Research, 1 (2), 45-50.

Shephard, R. J. (1988). PAR-Q Canadian Home Fitness Test and exercise screening alternatives. Sports Medine, 5(3), 185-195.

Silva, J. C. G., Neto, G. R., Freitas, E., Neto, E., Batista, G., Torres, M., \& Sousa, M. S. C. (2015). Chronic effect of strength training with blood flow restriction on muscular strength among women with osteoporosis. Journal of Exercise Physiologyonline, 18(4), 33-41.

Stachenfeld, N. S., Silva, C., \& Keefe, D. L. (2000). Estrogen modifies the temperature effects of progesterone. Journal of Applied Physiology, 88(5), 1643-1649.

Takano, H., Morita, T., Iida, H., Asada, K., Kato, M., Uno, K., . . . Hirata, Y. (2005). Hemodynamic and hormonal responses to a short-term lowintensity resistance exercise with the reduction of muscle blood flow. European Journal of Applied Physiology, 95(1), 65-73.

Vechin, F. C., Libardi, C. A., Conceição, M. S., Damas, F. R., Lixandrão, M. E., Berton, R. P. B., . . . Chacon-Mikahil, M. P. T. (2015). Comparisons 
40 | GR Neto, JS Novaes, AT Araújo Júnior, JCG Silva, RP Souza, MS Cirilo-Sousa

between low-intensity resistance training with blood flow restriction and high-intensity resistance training on quadriceps muscle mass and strength in elderly. Journal of Strength and Conditioning Research, 29(4), 1071-1076. doi: 10.1519/JSC.0000000000000703

Vera-Cala, L. M., Orostegui, M., Valencia-Angel, L. I., López, N., \& Bautista, L. E. (2011). Accuracy of the Omron HEM-705 CP for blood pressure measurement in large epidemiologic studies.
Arquivos Brasileiros de Cardiologia, 96(5), 393-398. doi: http://dx.doi.org/10.1590/S0066782X2011005000038

Vieira, P. J. C., Chiappa, G. R., Umpierre, D., Stein, R., \& Ribeiro, J. P. (2013). Hemodynamic responses to resistance exercise with restricted blood flow in young and older men. Journal of Strength and Conditioning Research, 27(8), 22882294. doi: 10.1519/JSC.0b013e318278f21f

All content of Journal Motricidade is licensed under Creative Commons, except when otherwise specified and in content retrieved from other bibliographic sources. 